\title{
Commercial blood cell separation systems versus tube centrifugation methods for the preparation of platelet-rich plasma: a preliminary cross-sectional study
}

\author{
Bibiana Trevisson-Redondo' (1), Ricardo Becerro-de-Bengoa-Vallejo² (1), \\ David Sevillano ${ }^{*}$ (1), Natalia González ${ }^{3}$ (1) , Marta Elena Losa-Iglesias ${ }^{4}$ (1), \\ Daniel López-López ${ }^{5}$ (D) Luis Alou ${ }^{3}$ (i)
}

\begin{abstract}
SUMMARY
OBJECTIVE: Clinical studies claim that platelet-rich plasma (PRP) accelerates tissue healing due to its high concentration of growth factors and that the combination with leukocytes improves the antimicrobial effect of the concentrate. Most of these studies obtained PRP using different separation systems, and few analyzed the content of the PRP used for treatment. This study characterized the composition of PRP and white blood cells (WBC) from a single donor produced by three commercially available PRP separation systems and two anticoagulated general analytical tubes.

METHODS: Five patients donated $50 \mathrm{~mL}$ of blood, which was processed to produce PRP and WBC using three PRP concentration systems (i.e., Easy PRP Kit, GloPRP, and Wego) and two tubes for general analysis anticoagulated with ethylenediaminetetraacetic acid (EDTA) and citrate. Platelets and WBC in combination with their concentrates were analyzed by automated systems in a clinical laboratory.

RESULTS: There were no significant differences in the average concentrations of PRP platelets and WBC between GloPRP and the tubes for general analysis with EDTA and citrate; however, the Easy PRP Kit gave results much superior to the rest of the methods, especially comparing it with the Wego Kit, whose concentrates were especially low, even nonexistent for WBC.

CONCLUSIONS: The Easy PRP Kit concentrates WBC-rich PRP, resulting in increased WBC concentrations, compared with low WBC-low PRP of GloPRP and general tube methods for EDTA and citrate analysis and the even lower concentration of PRP from the Wego Kit, with the absence of leukocytes.

KEYWORDS: Platelet-Rich Plasma. Leukocytes. Blood.
\end{abstract}

\footnotetext{
'Universidad de León, Faculty of Health Sciences, SALBIS Research Group, Nursing Department - León, Spain.

2Universidad Complutense de Madrid, School of Nursing, Physiotherapy and Podiatry, Nursing Department - Madrid, Spain.

${ }^{3}$ Universidad Complutense de Madrid, School of Medicine, Microbiology Section, Medicine Department - Madrid, Spain.

${ }^{4}$ Universidad Rey Juan Carlos, Faculty of Health Sciences - Madrid, Spain.

${ }^{5}$ Universidade da Coruña, Faculty of Nursing and Podiatry, Research, Health and Podiatry Group, Department of Health Sciences - Ferrol, Spain.

*Corresponding author: dsevill@ucm.es

Conflicts of interest: the authors declare there is no conflicts of interest. Funding: none.

Received on November 11, 2020. Accepted on November 27, 2020.
} 


\section{INTRODUCTION}

Platelet-rich plasma (PRP) is a blood product formed by concentrating platelets in a small volume of plasma and is used for a wide variety of applications ${ }^{1}$.

The mechanism of action of PRP is unknown; however, the release of high concentrations of growth factors from platelet alpha granules as platelets degranulate is believed to play a critical role ${ }^{2}$.

The PRP was first recognized as an effective agent for bone and tissue repair within the field of dentistry and oral maxillofacial surgery ${ }^{3}$. Its applicability then spread to the fields of plastic surgery by demonstrating an evidence of improved skin graft wound healing ${ }^{4,5}$.

A number of commercial systems are available that result in platelet products with a wide range of platelet and leukocyte concentrations, although there is limited information available regarding the optimal platelet and leukocyte contents necessary to achieve a desired biological effect, and it may be that specific products are better for certain applications ${ }^{6}$.

There is no single definition for PRP in the literature, and the "PRP" products include wide ranges of platelet and leukocyte concentrations that reflect the various separation methods and the lack of consensus on specific composition of the final product, and furthermore, platelet numbers are the primary concern, but optimal leukocyte concentrations in PRP is a topic of discussion in this study $y^{6,7}$.

Platelet concentrations in PRP commonly range from a 3to 5 -fold increase over whole blood (WB) or a minimum concentration of $300,000-1,000,000$ platelets $/ \mathrm{mL}^{8}$.

Therefore, the purpose of this study was to assess the concentration of platelets and white blood cells (WBC) of PRP produced by three new commercial systems and two tubes for general analytical tests, in order to verify if the kits reach the minimum concentration and if the tubes for general analytical tests are also capable of achieving it, or if, on the contrary, it is necessary to resort to the commercial systems created to alkalize the ideal concentrations, and the tubes for general analysis are not useful in order to concentrate platelets and WBC.

\section{METHODS}

\section{Participant recruitment}

Five healthy volunteers (i.e., two men and three women, aged 31-64 years) who were patients at the 2010 Podiatric Clinic, León, Spain were invited to participate in this study. Individuals were free from all chronic illnesses and not taking any regular medication, including aspirin and nonsteroidal anti-inflammatory drugs. Prior to recruitment, the objectives and procedures involved in this study were explained to participants, and each signed an informed consent form. All individuals fulfilled the inclusion criteria and consented to be included as participants in this study.

\section{Challenge PRP production methods and PRP preparation}

Blood $(60 \mathrm{~mL})$ was collected by venipuncture from the antecubital vein of each donor using a $21 \mathrm{G} \times 1.9 \mathrm{~cm}$ butterfly needle (BD Vacutainer, Franklin Lakes, NJ). WB was drawn into two plain 4.5-mL BD Vacutainer ethylenediaminetetraacetic acid (EDTA) tubes, two plain 4.5-mL BD Vacutainer sodium citrate (SC) 3.2\% tubes, and SC pre-filled syringes of the three commercial blood separation systems, namely, the classic PRP Kit (cl-PRP Kit) based on gel PRP tubes (Wego, Shenzhen, China), the Easy PRP Kit ${ }^{\circ}$ (Mesotech, Naples, Italy), and GloPRPÒ (Glofin, Ösalo, Finland) systems. An additional $2 \mathrm{~mL}$ of WB was collected into an EDTA tube to determine the basal count of platelets and leukocytes (WBC) in WB, including neutrophils, lymphocytes, and monocytes among WBC fractions (Echevarne Laboratory, HM San Francisco Hospital, León, Spain).

The PRP preparation technique was adapted from previously published methods ${ }^{9-11}$ and following the indications of the manufacturer's kits. All PRP protocols were based on a single-centrifugation step for 5-10 min (Nahita centrifuge, Ibor Médica, Spain), except for Glo-PRP system for which a double centrifugation process $(1,200 \times g$ for $5 \mathrm{~min}$ followed by $1,200 \times g$ for $10 \mathrm{~min}$ ) was used. After centrifugation, all tubes and systems were allowed to rest for $5 \mathrm{~min}$ to facilitate the settling of platelets onto the buffy coat. The PRP was collected using a needle attached to a $5-\mathrm{mL}$ syringe measuring $21 \mathrm{G} \times 40 \mathrm{~mm}$ under the naked eye visualization. With the tube stoppers removed, the needle tip was positioned so as to just touch the buffy coat. The syringe plunger was gently raised to vacuum up the platelets on the buffy coat, and the needle tip was slowly moved along the buffy layer ${ }^{12}$.

A total volume of $0.3-0.5 \mathrm{~mL}$ buffy coat was extracted from each tube into the collection syringe. A total PRP of 2.0-2.5 $\mathrm{mL}$ per participant was collected and transferred into an EDTA tube for analysis. The number of platelets and WBC present in the venous blood and the PRP were determined. The ratio of platelet levels in PRP to venous blood (PRP:WB ratio) was calculated to determine the ability of the current method to concentrate platelets and $\mathrm{WBC}^{12}$. The platelet and $\mathrm{WBC}$ analyses, both in the WB (baseline) and after centrifugation by the five methods of preparing PRP, were measured in milliliters.

\section{Statistical analysis}

The descriptive analysis of the characteristics of participants was performed. Continuous variables were reported using mean and standard deviation as well as lower and upper limits for a $95 \%$ 
confidence interval $(95 \% \mathrm{CI})$ or median and interquartile range (IQR). The categorical data were presented as frequencies and percentages. A repeated-measures one-way ANOVA followed by the Dunnett's multiple comparison test and ANOVA followed by the Tukey's multiple comparison test were performed to determine the differences in platelets and WBC contents between the WB and PRP obtained by each concentrator method and between systems used in this study, under the assumption that all the data suitably measured followed an approximately Gaussian distribution according to the Shapiro-Wilk normality test.

For all analyses, a value of $\mathrm{p}<0.05$ was considered statistically significant. The data obtained were analyzed using GraphPad Prism version 8.0.0 for Windows (GraphPad Software, San Diego, CA, USA).

\section{RESULTS}

Five healthy patients were recruited, according to the sample size of the previous studies ${ }^{6}$ with an average age of $47.40 \pm 15.58$ years (95\%CI $28.03-66.76$ years) and a body mass index of $28.79 \pm 4.81$ (95\%CI 22.82-34.77).

The platelet and WBC counts in WB and in the PRP obtained with each system are summarized in Table 1 . All methods, except the cl-PRP system, significantly increased WB platelet density (i.e., Easy PRP Kit and citrated tubes, $\mathrm{p}<0.0001$; EDTA tubes, $\mathrm{p}=0.001$; and the GloPRP system, $\mathrm{p}=0.011$ ). However, the mean increase in the platelet factor (PRP:WB ratio) provided by the Easy PRP Kit system was much greater (by 3.56 times versus 1.36-2.03 times basal platelets for Easy PRP Kit versus the other methods; $\mathrm{p}<0.05$ ) (Figure 1), reaching a significantly higher PRP platelet concentration than the other blood concentration methods. The platelet density achieved in the PRP was not significantly different between EDTA tubes, citrated tubes, GloPRP, or cl-PRP systems. The mean increase in the WBC factor was relatively less. The Easy PRP Kit system also showed the highest PRP:WB ratio (2.41 times versus $0.00-$ 1.13 times baseline WBC for Easy PRP Kit versus all other systems), reaching WBC densities significantly higher than WB and those obtained with any other system. The cl-PRP system did not concentrate WBC, and the densities reached by the rest of the systems were not significantly different from those measured in WB (Figure 1).

As shown in Figure 2, the concentration PRP:WB ratio for the different WBC fractions was markedly different among systems, being lower for neutrophils compared with lymphocytes and monocytes.

Table 1. Platelet and white blood cells count (cells $\times 10^{6} / \mathrm{mL}$ ) in whole blood and platelet-rich plasma obtained by different concentrator methods.

\begin{tabular}{|c|c|c|c|c|c|c|}
\hline & \multicolumn{3}{|c|}{ Platelets } & \multicolumn{3}{|c|}{ WBC } \\
\hline & Mean \pm SD & Median & \multirow{2}{*}{$p$} & Mean \pm SD & Median & \multirow{2}{*}{$p$} \\
\hline & $(95 \% \mathrm{Cl})$ & (IQR) & & $(95 \% \mathrm{Cl})$ & $(\mathrm{IQR})$ & \\
\hline \multirow{2}{*}{ WB } & $291.00 \pm 64.09 *$ & 304.00 & \multirow{2}{*}{0.81} & $8.02 \pm 2.12^{+}$ & 7.53 & \multirow{2}{*}{0.05} \\
\hline & $(211.43-370.57)$ & $(122.50)$ & & $(5.38-10.65)$ & (3.03) & \\
\hline \multirow{2}{*}{ Easy PRP Kit } & $1008.00 \pm 224.12^{\ddagger}$ & 1023.00 & \multirow{2}{*}{0.99} & $18.53 \pm 4.45^{\S}$ & 18.60 & \multirow{2}{*}{0.40} \\
\hline & $(729.33-1286.00)$ & $(398.52)$ & & $(13.01-24.05)$ & $(8.82)$ & \\
\hline \multirow{2}{*}{ EDTA tube } & $573.19 \pm 127.49$ & 582.14 & \multirow{2}{*}{0.99} & $8.72 \pm 2.09$ & 8.76 & \multirow{2}{*}{0.40} \\
\hline & (414.88-731.49) & $(226.63)$ & & $(6.12-11.32)$ & $(4.15)$ & \\
\hline \multirow{2}{*}{ Citrated tube } & $613.79 \pm 147.80$ & 670.95 & \multirow{2}{*}{0.51} & $8.28 \pm 1.99$ & 8.31 & \multirow{2}{*}{0.40} \\
\hline & $(430.28-797.31)$ & $(279.39)$ & & $(5.81-10.74)$ & $(3.94)$ & \\
\hline \multirow{2}{*}{ GloPRP system } & $468.97 \pm 104.31$ & 476.30 & \multirow{2}{*}{0.99} & $8.16 \pm 1.96$ & 8.20 & \multirow{2}{*}{0.40} \\
\hline & $339.45-598.49$ & $(185.43)$ & & $(5.73-10.60)$ & (3.89) & \\
\hline \multirow{2}{*}{ Cl-PRP } & $384.61 \pm 85.55$ & 390.62 & \multirow{2}{*}{0.99} & $0.0 \pm 0.0^{\prime \prime}$ & 0.0 & \multirow{2}{*}{-} \\
\hline & (278.39-490.83) & $(152.07)$ & & $(0.0-0.0)$ & $(0.0)$ & \\
\hline
\end{tabular}

${ }^{*} p<0.0001$ versus Easy PRP Kit and citrated tube, $p=0.001$ versus EDTA tubes and $p=0.011$ versus GloPRP system; ${ }^{\dagger} p<0.0001$ versus Easy PRP Kit and cl-PRP; ${ }^{\ddagger} p<0.0001$ versus GloPRP and cl-PRP, $p=0.0011$ versus EDTA tube and $p=0.0031$ versus citrated tube; ${ }^{\S} p<0.0001$ versus all methods; / $p \leq 0.0005$ versus EDTA tube, citrated tube, and GloPRP.

SD: standard deviation; Cl: confidence interval; IQR: interquartile range; p: Shapiro-Wilk test with a statistical significance for a p-value $<0.05$, with a 95\%Cl; WB: whole blood; PRP: platelet-rich plasma; EDTA: ethylenediaminetetraacetic acid. 


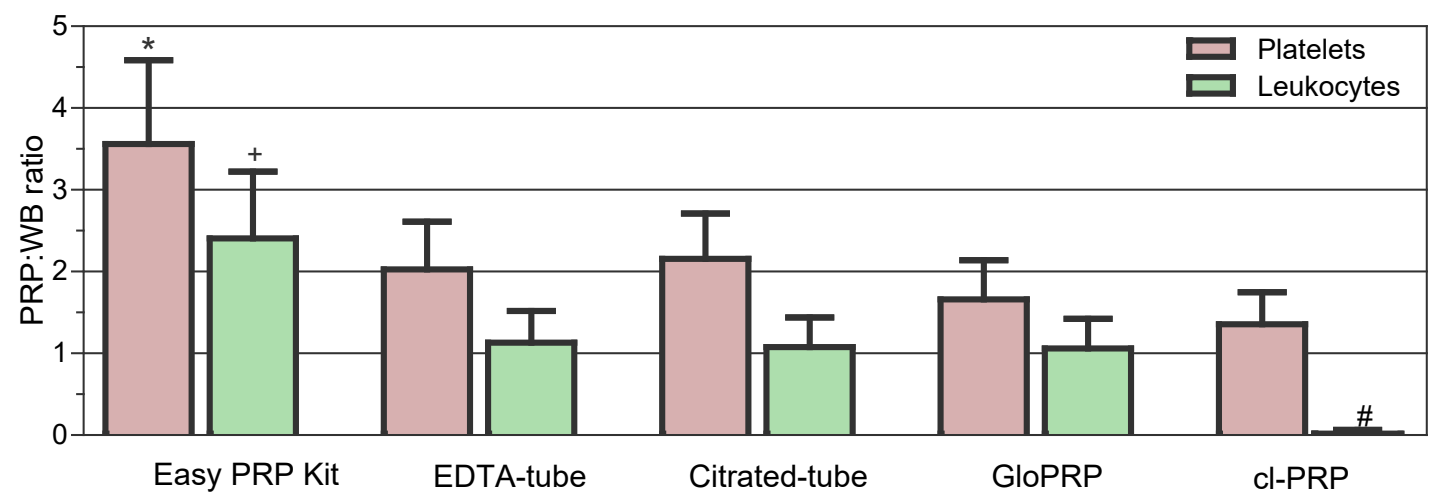

" $p \leq 0.0192$ versus all methods. ${ }^{+} p \leq 0.0026$ versus all methods. " $p \leq 0.013$ versus EDTA tube, citrated tube, and GloPRP.

WBC: white blood cells; WB: whole blood; PRP: platelet-rich plasma; SD: standard deviation; EDTA: ethylenediaminetetraacetic acid.

Figure 1. Fold change of platelets and white blood cells versus basal whole blood (platelet-rich plasma:whole blood ratio) in the concentrates obtained by each blood separation method (meantstandard deviation). Platelet-rich plasma:whole blood ratio of 1 ; no cell concentration.

A

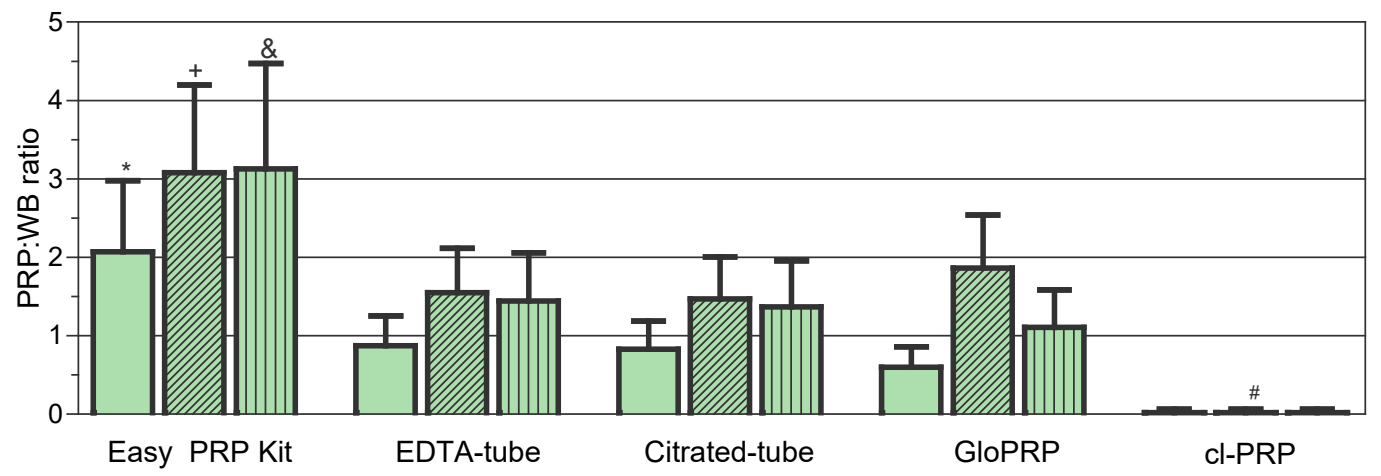

B

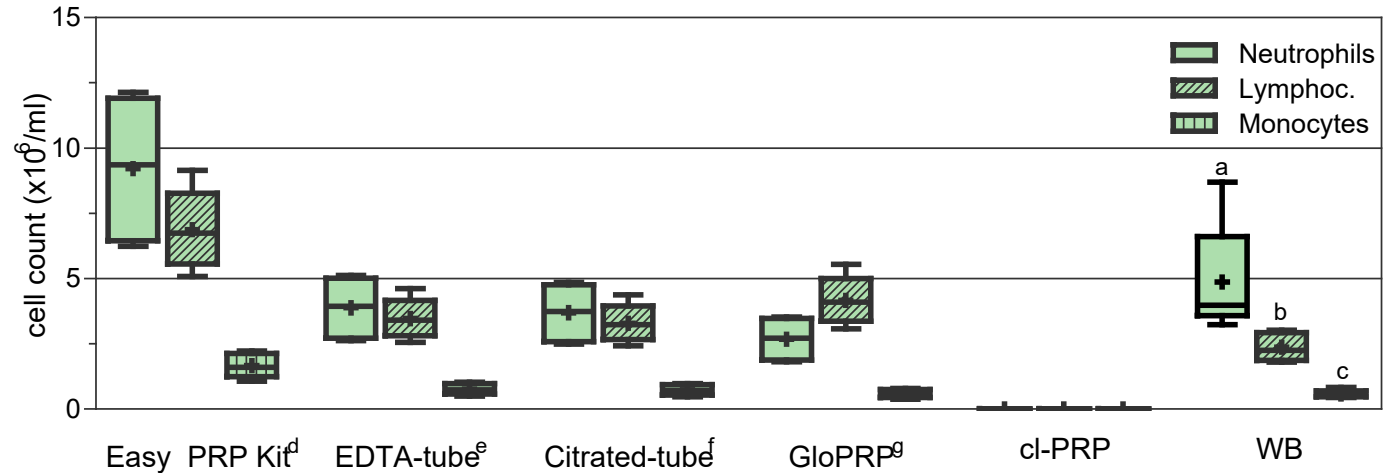

" $p \leq 0.0065$ versus all methods; ${ }^{+} p \leq 0.0148$ versus EDTA tube, citrated tube, and cl-PRP. ${ }^{\circledR} p \leq 0.014$ versus EDTA tube, citrated tube, and cl-PRP. ${ }^{\#} p \leq 0.0195$ versus EDTA tube, citrated tube, and GloPRP. ${ }^{a} p<0.0001$ versus Easy PRP Kit and cl-PRP, $p=0.033$ versus GloPRP; ${ }^{b} p<0.0001$ versus Easy PRP Kit and cl-PRP, $p=0.0024$ versus GloPRP; ${ }^{c} p<0.001$ versus Easy PRP Kit and cl-PRP; ${ }^{d} p \leq 0.0014$ versus all methods for WBC fractions; ${ }^{e} p \leq 0.0036$ versus $\mathrm{Cl}$-PRP for WBC fractions; ${ }^{\mathrm{f}} \mathrm{p} \leq 0.0058$ versus $\mathrm{Cl}-\mathrm{PRP}$ for WBC fractions; ${ }^{9} \mathrm{P} \leq 0.013$ versus $\mathrm{cl}$-PRP for lymphocytes and monocytes. WBC: white blood cells; WB: whole blood; PRP: platelet-rich plasma; EDTA: ethylenediaminetetraacetic acid.

Figure 2. The efficiency of the different blood separation methods to concentrate neutrophils, lymphocytes, and monocytes. (A) Platelet-rich plasma:whole blood ratio. (B) Box plot (minimum to maximum) of cell count $\left(\times 10^{6} / \mathrm{mL}\right)$ recovered from each concentrate (median: line in the middle of the box; mean: + ). 


\section{DISCUSSION}

In this study, we demonstrated a general difference in the platelet factor increase and platelet recovery rates in the PRP concentrated by the commercial Easy PRP Kit and the remaining PRP preparing methods, which was a surprising finding given that the starting blood volume (i.e., $11 \mathrm{~mL}$ for Easy PRP Kit, $9 \mathrm{~mL}$ for GloPRP and the cl-PRP Kit, and $8 \mathrm{~mL}$ for EDTA and citrate general analytical tubes) and the centrifugation patterns (i.e., rotation force and the number of centrifugation steps) used were similar to other methods used or even more unfavorable (i.e., GloPRP used twostep centrifugation procedure to prepare $\mathrm{PRP}$ ), producing equal volumes of PRP $(2 \mathrm{~mL})$. However, both arguments alone do not explain the performance of Easy PRP Kit system, since with the increase of only $3 \mathrm{~mL}$ of blood volume, it concentrated between 40 and $60 \%$ more platelets than the others, and the centrifugal force accommodated was identical for different methods. Thus, based on the recent theoretical predictions by Piao et al. to establish the optimal conditions for PRP preparation, we believed that both the geometry of the device and the use of relatively short spinning times (4-5 min) play an important role in the recovery rates of the platelets ${ }^{13}$. However, it is important to note that the increase in the platelet factor for the Easy PRP Kit system in this study (3.56 times the basal platelet count) was far from the system efficiency displayed by the manufacturer (7-9 times). Consequently, the skill of the clinician and the familiarity with the use of the device are the essential factors to predict the platelet capture efficiency of the different PRP separation systems in the clinical setting and, thus, to be able to satisfactorily advise patients on the PRP content provided by each one of them.

Interestingly, both tube-based methods, which achieved between 56 (EDTA) and 60\% (SC) of the total platelets concentrated by the Easy PRP Kit system, exhibited a higher effciency than the commercial GloPRP systems (i.e., 46\% of the total platelets recovered by Easy PRP Kit), which included a two-step centrifugation process, or the cl-PRP Kit (38\%) to concentrate platelets. These results confirm that the anticoagulant incorporated into the system modifies the efficiency of the separation and concentration of the blood fractions as previously described by Amaral et al. ${ }^{14}$ Furthermore, since all systems were anticoagulated with SC, the use of EDTA as anticoagulant would result in a particularly low platelet recovery rate for the GloPRP and the classic PRP Kit.

In this study, we also found a significant difference in the WBC obtained by each blood separation method. The Easy PRP Kit system again demonstrated the highest increase in the WBC factor, achieving WBC densities significantly higher than those measured in WB. In contrast, leukocytes in the concentrates obtained by the blood tube collection-based methods or by GloPRP produced virtually no increase from WB levels.
This study revealed that regardless of the specific efficiencies of each system, the cell concentration factor was blood cell size dependent; the smaller the blood cell size, the greater the concentration factor. Apart from platelets, highly concentrated based on the size, the concentration factor among leukocytes was higher for lymphocytes $>$ monocytes $>$ neutrophils. Neutrophils, along with monocytes/macrophages, are the effectors of the expected innate immune response of the WBC fraction and have a greater representation in WB and higher microbicidal activity in comparison ${ }^{15}$. Thus, this cell size-based efficiency, which implies an overrepresentation of lymphocyte fraction in the WBC extract, would be suitable for our therapeutic expectations with WBC if methods with poor efficiency in WBC concentration, such as EDTA or SC tubes or GloPRP system, were used. These methods significantly concentrated lymphocytes, but decreased the basal neutrophil concentration in WB. For this reason, if we are truly interested in the clinical use of platelets in combination with "leukocytes," it would be highly recommended to know in advance the concentration power of the system for the different WBC fractions.

\section{CONCLUSION}

The Easy PRP Kit concentrates WBC-rich PRP, resulting in increased WBC concentrations, compared with low WBClow PRP of GloPRP and general tube methods for EDTA and citrate analysis and the even lower concentration of PRP from the Wego Kit, with the absence of leukocytes.

\section{AUTHORS' CONTRIBUTIONS}

BTR: Data curation, Formal analysis, Investigation, Writing - review \& editing. RBBV: Conceptualization, Formal analysis, Writing - original draft, Writing - review \& editing. DS: Conceptualization, Data curation, Formal analysis, Investigation, Methodology, Writing - original draft, Writing - review \& editing. NG: Data curation, Formal analysis, Investigation, Methodology, Writing - review \& editing. MELI: Conceptualization, Writing - original draft, Writing - review \& editing. DLL: Data curation, Formal analysis, Investigation, Writing - review \& editing. LA: Conceptualization, Data curation, Formal analysis, Investigation, Methodology, Writing - original draft, Writing - review \& editing.

\section{ETHICS STATEMENT}

The Research Ethics Committee of the Rey Juan Carlos University of Madrid approved this study. 


\section{REFERENCES}

1. Sampson S, Gerhardt M, Mandelbaum B. Platelet rich plasma injection grafts for musculoskeletal injuries: a review. Curr Rev Musculoskelet Med. 2008;1(3-4):165-74. https://doi. org/10.1007/s12178-008-9032-5

2. Anitua E, Andia I, Ardanza B, Nurden P, Nurden AT. Autologous platelets as a source of proteins for healing and tissue regeneration. Thromb Haemost. 2004;91(1):4-15. https:// doi.org/10.1160/TH03-07-0440

3. Grageda E. Platelet-rich plasma and bone graft materials: a review and a standardized research protocol. Implant Dent. 2004;13(4):301-9. https://doi.org/10.1097/01. id.0000148555.91063.06

4. Man D, Plosker H, Winland-Brown JE. The use of autologous platelet-rich plasma (platelet gel) and autologous plateletpoor plasma (fibrin glue) in cosmetic surgery. Plast Reconstr Surg. 2001;107(1):229-37; discussion 238-9. https://doi. org/10.1097/00006534-200101000-00037

5. Garrido-Castells X, Becerro-de-Bengoa-Vallejo R, CalvoLobo C, Losa-Iglesias ME, Palomo-López P, Navarro-Flores $E$, et al. Effectiveness of leukocyte and platelet-rich fibrin versus nitrofurazone on nail post-surgery bleeding and wound cicatrization period reductions: a randomized single blinded clinical trial. J Clin Med. 2019;8(10):1552. https:// doi.org/10.3390/jcm8101552

6. Castillo TN, Pouliot MA, Kim HJ, Dragoo JL. Comparison of growth factor and platelet concentration from commercial platelet-rich plasma separation systems. Am J Sports Med. 2011;39(2):26671. https://doi.org/10.1177/0363546510387517

7. McLellan J, Plevin S. Does it matter which platelet-rich plasma we use? Equine Veterinary Education. 2011;23(2):101-4. https://doi.org/10.1111/j.2042-3292.2010.00185.x

8. Liu Y, Kalén A, Risto $O$, Wahlström $O$. Fibroblast proliferation due to exposure to a platelet concentrate in vitro is $\mathrm{pH}$ dependent. Wound Repair Regen. 2002;10(5):336-40. https:// doi.org/10.1046/j.1524-475X.2002.10510.x

9. Rutkowski JL, Thomas JM, Bering CL, Speicher JL, Radio NM, Smith DM, et al. Analysis of a rapid, simple, and inexpensive technique used to obtain platelet-rich plasma for use in clinical practice. J Oral Implantol. 2008;34(1):25-33. https:// doi.org/10.1563/1548-1336(2008)34[25:AAOARS]2.0.CO;2

10. Peterson NS, Reeves KD. Efficacy of one day training in low-cost manual preparation of high cellular platelet rich plasma. Journal of Prolotherapy. 2014;6:e922-e927. Available from: https://www.journalofprolotherapy.com/pdfs/volume_06/ volume_06_01_platelet_rich_plasma.pdf

11. Nagata MJH, Messora MR, Furlaneto FAC, Fucini SE, Bosco $A F$, Garcia VG, et al. Effectiveness of two methods for preparation of autologous platelet-rich plasma: an experimental study in rabbits. Eur J Dent. 2010;4(4):395-402. https://doi. org/10.1055/s-0039-1697859

12. Hamid MSA. Cost effectiveness of a platelet-rich plasma preparation technique for clinical use. Wounds. 2018;30(7):18690. PMID 30059343

13. Piao $\mathrm{L}$, Park $\mathrm{H}$, Jo $\mathrm{CH}$. Theoretical prediction and validation of cell recovery rates in preparing platelet-rich plasma through a centrifugation. PLoS One. 2017;12(11):e0187509. https:// doi.org/10.1371/journal.pone.0187509

14. Amaral RJFC, Silva NP, Haddad NF, Lopes LS, Ferreira FD, Bastos Filho $\mathrm{R}$, et al. Platelet-rich plasma obtained with different anticoagulants and their effect on platelet numbers and mesenchymal stromal cells behavior in vitro. Stem Cells Int. 2016;2016:7414036. https://doi.org/10.1155/2016/7414036

15. Kumar KP, Nicholls AJ, Wong CHY. Partners in crime: neutrophils and monocytes/macrophages in inflammation and disease. Cell Tissue Res. 2018;371(3):551-65. https://doi.org/10.1007/ s00441-017-2753-2 